ANNIKA WaLLIN

\title{
THE EVERYDAY OF DECISION-MAKING'
}

In this paper I will argue for the importance of studying the everyday of decision making, if we want to know more about what decision processes look like, how rational human beings are, and how we (if necessary) can improve decision making.

\section{Decision making without the everyday}

When issues such as rationality and decision making are discussed, we often go back to standard decision theoretic descriptions of decision making, leading to recommended strategies such as the Principle of Maximizing Expected Utility. In order to use this principle we have to have access to at least two courses of action that lead to different outcomes. Savage (1954) describes this situation by a chef cooking an omelet. At the time of the decision this person has broken five good eggs into a bowl, but a sixth one is available. For some reason, this egg has to be used now or thrown away (perhaps the kitchen is closing for the summer?). Savage describes the situation in the following way:

\begin{tabular}{|l|l|l|}
\hline \multirow{2}{*}{ Act } & \multicolumn{2}{|c|}{ State } \\
\cline { 2 - 3 } & \multicolumn{1}{|c|}{ good } & \multicolumn{1}{c|}{ rotten } \\
\hline Break into bowl & Six-egg omelet & $\begin{array}{l}\text { No omelet, and five good eggs } \\
\text { destroyed }\end{array}$ \\
\hline Break into saucer & Six-egg omelet and a saucer to wash & Five-egg omelet and a saucer to wash \\
\hline Throw away & $\begin{array}{l}\text { Five-egg omelet and a good egg } \\
\text { destroyed }\end{array}$ & Five-egg omelet \\
\hline
\end{tabular}

1 This paper is partly based on the chapter "Decision making in everyday life" (Wallin, 2008), but has been updated and changed. 
In order to decide upon a course of action the chef will have to decide how good or bad each possible outcome is. This is done by assessing the utility of a sixegg omelet, the same omelet with a saucer to wash, no good omelet and five good eggs destroyed, and so on. In addition the decision maker will have to have an idea of how probable it is that the egg is rotten. Once this is done, the expected utility for each possible action can be determined. For instance, the chef who thinks the probability of a rotten egg is low, and hates waste will probably break the egg directly into the bowl, whereas a cautious and hungry person will use the saucer. Bayesian decision theory states that a decision maker should always select the alternative with the highest expected utility. This is the Principle of Maximizing Expected Utility.

It is not entirely clear from the Principle of Maximizing Expected Utility how decision makers should proceed when making a decision. Should they make lists, like the one above, or do they have other options? The reason that Bayesian decision theory is relatively mute on the specifics of decision-making is that it is a normative theory. It describes what a good decision looks like, but does not presume anything about how decisions are made. Nevertheless, the most obvious option for someone attempting to maximize expected utility is to identify each possible action and state of the world, to assign each state a probability and each possible outcome an utility. But although the recommendation to do so seems very sensible, it is not altogether easy to follow. As a matter of fact, the theoretical framework developed by Savage requires an agent that has, among other things, full information and infinite sensitivity. This is obviously not true for any living decision maker, but the intention of models of ideal rational behaviour (such as Savage's) is not to be realistic, but rather to explore the nature of rational choice (cf. Sahlin et al., 2010; Wallin, 2013).

\section{Decision making without the everyday in judgment and decision-making}

Interestingly, however, the ideal rational model (often named Economic man) has had a strong presence in the area of judgment and decision making despite the fact that it is obviously unrealistic. This is not entirely unsurprising: after all it is a model of rational decision making, and alternative models are scarce. Ward Edwards explicitly introduced Economic man into the field of judgment and decision-making in the 1950s: 
It is easy for a psychologist to point out that an Economic man who has the properties discussed above [complete information, infinite sensitivity, rationality] is very unlike a real man. In fact, it is so easy to point this out that psychologists have tended to reject out of hand the theories that result from these assumptions. This isn't fair. [... The most useful thing to do with a theory is not to criticize its assumptions but rather to test its theorems. (Edwards, 1954: 382)

This approach was picked up by Amos Tversky and Daniel Kahneman's heuristics and biases research. Perhaps it culminated with a Science paper (Tversky, Kahneman, 1974), in which violations of some very fundamental "statistical rules" were summarized and described (ibid.: 1130). Among other things, participants did not seem sensitive to the prior probability of an outcome, to sample size, or to predictability. Through such deviations the authors argued for the existence of heuristics: simple decision rules described as being "highly economical and usually effective", but leading to "systematic and predictable errors" (ibid.: 1131). These, and similar results, have been famously described as having "bleak implications for human rationality" (Nisbett, Borgida, 1975: 935). How the results should be interpreted, whether participants violate fundamental statistical rules, and whether the implications for human rationality are truly bleak has been discussed since. The resulting debate has been named the rationality wars by Richard Samuels and colleagues (2002).

I will not focus on the rationality wars here, nor on the issue of how rationality has to be studied. I will simply point out that the research traditions that aim at studying how closely human behaviour approximates Economic man has a focus on using paper and pencil tasks where, for instance, sensitivity to prior probabilities is examined. This leads to famous and extremely well investigated tasks involving taxicabs, feminist bank tellers and Asian diseases that have little resemblance to the type of decisions and judgements we usually are engaged in. It is my strong belief that this research has to be complimented by studies of real life decision-making and this for three reasons. First, if our decision-making is shaped by anything, it is by common activities for which we receive feedback. Therefore, the decisions strategies shaped by, for instance, the tasks we accomplish in a supermarket, are more likely to influence our decision making regarding complex issues such as choice of medical treatments, voting behaviour and economic commitments than the other way around. Second, if we study familiar tasks we will get a more realistic, useful and likely positive view of human rationality. Thirdly, the study object - the tasks with which decision making is usually 
studied, is likely to have a great impact on what the resulting cognitive models are. In this I wholeheartedly agree with Ward Edwards when he says: "My own guess is that most successful models now available are successful exactly because of their success in describing tasks, not people" (Edwards, 1971: 640).

\section{Why is it important to focus on the everyday?}

Looking at real life decision situations it is obvious that decision makers do not compute the expected utility of each possible choice, as has been suggested by decision theoretical approaches to decision making. The important question, in my mind, then becomes how decisions are made - in real life settings - and in general.

One way in which my colleagues and I have tried to understand this is by investigating consumer behaviour in stores. By approaching people that do their everyday shopping we get a completely different decision task; a task that is familiar, where people are motivated, and where they over time receive feedback on the trade off between effort and decision outcomes.

In one of our studies we observed shoppers buying (among other things) jam in a supermarket. These consumers had a choice of 91 different types of jam, all differing in taste, size, price, land of origin etc. in up to at least 90 different attributes. Despite this plethora of information, the average participant spent only approximately half a minute contemplating his or her choice (Gidlöf et al., 2013b). If Bayesian decision theory is interpreted as a recommendation to compute the expected utility of every possible jar of jam, and to select the one that best satisfies the decision makers' demands, grocery shopping would literally take forever. This state of affairs has been obvious to all researchers on decision-making, but regardless of this fact, the Principle of Maximizing Expected Utility was for a long time the only available description of how decisions are made. It was so ingrained in our way of thinking that researchers have spent decades trying to prove that it does not describe decisions in everyday life (see above). But if people do not list utilities and probabilities when they make a decision, what do they do? Can less taxing decision-making strategies still be reasonably successful? The answer is yes, but as we will see, decision quality then depends on the situation in which it is made. This is why I think that everyday decision-making is important.

So then, what do consumers do it if they do not maximize expected utility?

\section{2 \&) COGNITIVE SCIENCE}


One possibility is that the consumers bought the same kind of jam they ordinarily prefer, not even trying to update their knowledge about the other available kinds. Such a strategy saves time, and given that the shoppers are happy with their default choice, it is bound to be reasonably successful. Note however that these consumers run the risk of ignoring an even better option - perhaps a jam similar to their preferred one in all respects except a lower price. Thus a shopper sticking with what he or she knows is willing to trade an optimal choice for a quick, sufficiently good one. Herbert Simon (1956) called this principle of trying to find something "good enough" satisficing.

There are many strategies of this type. Decision makers can, for instance, use the strategy elimination by aspects: first compare all jams with respect to one of their attributes, such as their price. If one jar is cheaper than the rest, pick it. If several jars remain, compare these (and only these) with respect to another attribute, such as how much fruit they contain. Continue searching through the attributes in this way until you are left with only one jar of jam (Tversky, 1972). It is of course possible that there is another jar of jam somewhere on the shelf that has a better combination of attributes than the one you picked. Thus, a consumer eliminating by aspects is also satisficing. They trade the cost of not finding the best possible option for the gain of not having to spend too much time and energy searching for the jam they buy. Such strategies are generally quicker and less demanding than comparing the utility of each option.

It is clear that people use relatively little time and information in most everyday decisions. To remain within the supermarket setting, Hoyer (1984) observed consumers buying detergent and found that they spent on average 13 seconds in the isle. When they were approached and interviewed about their decisions an overwhelming majority (90\%) gave merely one reason for their choice such as its price, or their experience with the products' performance. That consumers pay little attention in such everyday decisions is further confirmed by the fact that if you approach consumers just after they have selected a product not even half of them can correctly estimate its price, and a fifth cannot even give a rough estimate (Dickson, Sawyer, 1990). It is highly unlikely that these decisions were made according to Savage's recommendations. The consumers' behaviour is far more similar to Simon's satisficers.

A decision maker using a relatively easily applied decision rule may also get additional advantages. There is some evidence that a decision that is too complex decreases in quality, both with respect to how satisfied and confident decision 
makers are with their choice and with respect to how likely they are to actually decide. One way in which this can happen is if decision makers are given too much information. This phenomenon has been given several different names over the years: information overload, choice deferral, the too much choice effect, and the paradox of choice. A particularly elegant demonstration of how complexity negatively affects decision makers is a study by the psychologists Sheena Iyengar and Mark Lepper (2000). They placed a tasting booth at an upscale supermarket where shoppers could stop and sample a variety of exotic jams. Some days the variety was larger, so that 24 types of jams were on offer. Other days only a subset of 6 of these 24 jams could be tasted (selected so that it contained the 2 most and least popular jams, and two jams of medium popularity). The shoppers were about twice as likely to try some of the 24 jams than they were to do so with the smaller set. But far more of those that stopped and sampled from the small selection actually bought a jar of jam.

One way this phenomenon has been explained is that it is too difficult to make a choice when the variety is large. A consumer choosing from a large variety will be confronted with more options, and someone trying to make an informed choice will thus have to consider more information. As a matter of fact, just telling people more about the properties of different options - such as the qualities of a particular jam - is enough to make choice more difficult and sometimes too difficult (Malhortra, 1982). Naturally the more information a decision rule requires, the more affected will the decision maker be when the amount of information increases. Thus it is possible that decision makers that rely on rules that require little information are less affected by the too much choice effect, that is, when they have to choose among many options, or are given lots of information about these options.

On the other hand it is clear that a decision maker that ignores information runs the risk of making a bad choice in the sense that $\mathrm{s} /$ he misses an even better opportunity. How worried should we be by this possibility? How much do decision makers loose by satisficing instead of optimizing? Even a satisficing decision maker may end up with the best possible option, however that is defined. To determine how damaging it is to ignore information we have to consider also the environment in which a decision is made. For instance, simulations reveal that a decision maker looking at a single most important attribute - say, price in the case of grocery shopping - is still highly likely to select one of the best available options (within $90 \%$ of the best possible value) if the environment is such that

\section{4 \&) COGNITIVE SCIENCE}


attractive features such as low price and a high content of fruit are positively correlated (Fasolo et al., 2007). For decisions in which positive attributes co-vary, decision makers that ignore all information except that pertaining to a single subjectively important attribute will still make highly successful predictions. When positive attributes do not co-vary, ignoring information may lead to worse outcomes. Thus an important aspect of decision-making is to select strategies that fit the environments in which they are used. Research in judgment and decision making indicate that people's decision strategies vary with types of tasks and decision environments (see for instance: Payne et al., 1993; Gigerenzer et al., 1999), and we know from our research that consumers in supermarkets are moderately successful (Gidlöf et al., 2013a). This leaves us with an important research questions: which strategies work well in which decision environments? (later on we will also ask how different decision strategies are activated).

\section{Which strategies work well in which decision environments?}

Decision strategies used in everyday life have to be frugal (i.e. require little information) and fast. It is impossible for a shopper facing almost a hundred types of jam to process all information carefully. Does this mean that we are bound to make bad choices? Actually not. Depending on the decision environment, fast and frugal decision strategies can be as successful as more complex strategies. They can even sometimes do better.

Imagine, for instance, a group of American and German students trying to determine for long lists of randomly selected pairs of cities, which is the larger one. Is Dortmund larger than Munich or vice versa? And what about San Diego and Sacramento? When actual students are given the same choices a remarkable thing happens: the American students make equally good predictions for German cities as they do for American ones (Gigerenzer, Goldstein, 1996; Goldstein, Gigerenzer, 1999; 2002). How can this be?

The answer appears to be that ignorance is not random. Students, and people in general, tend to recognize cities that are larger. Gigerenzer and Goldstein compared the number of mentions German cities got in the New York Times, and American cities in Die Zeit, and found a generally strong correlation between number of mentions and population size. If recognition is mediated by, for 
instance, mentions in the press, the ecological validity of recognition is, in this case, quite high. This means that a relatively ignorant student can utilize the non-random character of his or her ignorance when making a choice. Students that are familiar with both cities cannot, and have to rely on other information, such as the fact that Sacramento is the state capital of California. Such knowledge can be misleading (San Diego is actually bigger) and in such circumstances, knowing less can outperform knowing more. Ignorant people can make better choices - when the environment is right.

The recognition experiment demonstrates that it is sometimes an advantage to be ignorant. But there is other evidence that simple decision rules, such as taking the city you recognize (the recognition heuristic), may do well. Czerlinski and colleagues (1999) constructed 20 decision environments in which decision makers (in this case simulations following pre-defined decision rules) could search information about pairs of options and pick their winner, just as in the city example above. They were, for instance, required to predict dropout rates at high schools in Chicago given information about such things as their proportion of low-income students, non-white students and SAT scores. Another task was to predict the selling prices of houses in Pennsylvania based on information such as property taxes, garage space and total living space. These decision environments were based on statistics textbooks and reports, and were as true to real life as they could be. The authors constructed different rules that could be used to predict which house would be more expensive or which high school would have fewer dropouts. Some were relatively complicated such as multiple regressions, and some added the pros and cons without assigning them any weights (Dawes' rule). Other rules were even more simple, such as a rule that basically searched information in the order of its validity and made a decision as soon as one piece of information differentiated between the options. Since the rule searches information in order of its validity, that is, how accurate a decision based on this information will be, the rule was called take-the-best (note its similarity to elimination-by-aspects). The decision rules were then allowed to train on half of the available data sets (setting weights, or learning the validity of the different pieces of information as best as they could), and to make predictions for the other half. In this task the take-thebest-rule surprisingly outperformed both multiple regression, and Dawes' rule. A rule that ignored most of the available information thus made better predictions than rules that took all of it into account. How can this be? The most probable explanation is that the more complicated rules take information into consideration

\section{6 \&) COGNITIVE SCIENCE}


that does not help them make correct predictions. They take too much of the information in the training set into account - and "overfit" to circumstances that do not generalize ( $c f$. Martignon, Hoffrage, 1999).

The conclusion to draw from the above is that sometimes using less information can be better - when the environment is such that it pays off. Nobel laureate Herbert Simon put it in the following way: "Human rational behavior is shaped by a scissors whose two blades are the structure of the task environment and the computational capabilities of the actor" (1990: 7). It is clear that most human endeavours (and in particular everyday decision-making) have to use limited time and resources. The fact that simple decision rules can perform so well thus gives us good reasons to assume that it is these types of strategies that people use in their day-to-day activities. Whether this intuition is true will have to be, and is currently, tested in experimental studies. They will not be covered here. Instead the next question we will turn to is how people recognize which environment they are in.

\section{When are different decision rules activated?}

Oppenheimer (2003) challenged Gigerenzer and Goldstein's recognition heuristic by constructing pairs of cities in which some where well-known and small (such as Chernobyl and Los Alamos) whereas others did not exist, but sounded like they were situated in densely inhabited areas (such as, Al Ahbahib, Weingshe, and Las Besas). Not surprisingly, participants were more likely to guess that the non-existing (and therefore not recognized) cities were larger. In a way this is not surprising, since these pairs of cities were explicitly constructed to recognitions' disadvantage. However it points to an important question: what determines whether a decision rule is activated or not?

One answer could lie in adaptation. With time and experience decision makers learn that a particular strategy is generally successful in a certain type of settings (e.g. Payne et al., 1993; Gigerenzer et al., 1999). Such an adaptation can occur at both an individual and a cultural level (Wallin, 2007). Cultural and individual adaptation can also explain how particular decision rules could function unproblematically in relatively unknown environments. For instance, decision makers use possible causal relations between cues and criteria (the available information, and the outcome to be predicted) as a proxy for the ecological validity of each cue (Garcia-Retarmero et al., 2007). In this way, a rule such as take-thebest can be used also for environments in which cue validity is unknown. 
Another answer is that we often have no choice. Given the type of situation we are in, the time, information and computational resources available for a decision may be small, and thus force us to use a relatively fast a frugal decision rule. A wonderful example of this is a study by Ap Dijksterhuis and colleagues (2006). They presented participants with more or less complex decisions, such as choosing a house or a car. One group of participants were given time to think about their choice, whereas others were prevented from conscious deliberation through a distractor task. Those that were given time to think about their choices were less likely to pick the best out of four cars when the choice was complex (i.e. the options had 24 attributes). When the choice was not complex (the options had only 4 attributes) those that had thought about their choice did better. Dijksterhuis and associates explain this by the advantages of unconscious processing. Another explanation, that better fits the theme of this chapter, is that even if the environment determines that little time will be spent on a decision, reducing the information that is consciously processed in order to reach it might improve decision-making.

Naturally there are also differences between people in how much time they spend on a decision, or which decision rules they use. There have been attempts to measure this as a personality trait with questionnaires such as "need for cognition". Dijksterhuis and colleagues complemented their laboratory studies with one in real life. They looked a choices made in two different actual stores. One of them offers relatively complex choices (IKEA) whereas the other sells more simple products such as clothing accessories (Bijenkorf). Costumers were approached as they exited the store and were asked to fill in a questionnaire aimed at identifying consumers who had thought a lot, or relatively little about their choice. Some weeks later the customers were contacted and asked how happy they were with their choice. Non-thinking consumers were generally more happy with their complex choices, than thinking consumers were, and the opposite effect was found for the less complex products.

\section{Summary}

The goal of this chapter has been to show that everyday decision making matters. It is not enough to construct formal, normative, models of how decisions should be made, because these models are difficult to apply to real life deci- 
sion tasks. Furthermore, the environments in which decisions are made to a large extent determine the success of different decision rules. Given the right environment, ignorance can even outperform knowledge. There are many, highly useful, shortcuts to good decision making, such as exploiting ignorance, imitating others, or ignoring less important information. Such decision strategies can only be fully understood if we study the environments of everyday reasoning.

\section{References}

Czerlinski, J., Gigerenzer, G., Goldstein, D. (1999). The recognition heuristic: How ignorance makes us smart. In: G. Gigerenzer, P.M. Todd, The ABC Group, Simple heuristics that make us smart (pp. 97-118). New York: Oxford University Press.

Dickson, P.R., Sawyer, A.G. (1990). The price knowledge and search of supermarket shoppers. Journal of Marketing 54: 42-53.

Edwards, W. (1954). The theory of decision-making. Psychological Bulletin 51 (4): 380417.

Edwards, W. (1971). Bayesian and regression models of human information processing - a myopic perspective. Organizational Behavior and Human Performance 6: 639-648.

Dijksterhuis, A., Bos, M.W., Nordgren, L.F., van Baaren, R.B. (2006). On making the right choice: The deliberation-without-attention effect. Science 311: 1005-1007.

Fasolo, B., McClelland, G., Todd, P.M. (2007). Escaping the tyranny of choice: When fewer attributes make choice easier. Marketing Theory 7: 13-26.

Garcia-Retarmero, R., Wallin, A., Diekmann, A. (2007). Does causal knowledge help us be faster and more frugal in our decisions? Memory and Cognition 35 (6): 1399-1409.

Gidlöf, K., Wallin, A., Dewhurst, R., Holmqvist, K. (2013a). Using eye-tracking to trace a cognitive process: Gaze behavior during decision making in a natural environment. Journal of Eye Movement Research 6 (1): 1-14.

Gidlöf, K., Wallin, A., Mögelvang-Hansen, P., Holmqvist, K. (2013b). Material distortion of economic behavior and everyday decision making. Journal of Consumer Policy 36 (4): 389-402.

Gigerenzer, G., Goldstein, D. (1996). Reasoning the fast and frugal way: Models of bounded rationality. Psychological Review 98: 648-704.

Gigerenzer, G., Todd, P.M., The ABC Group (1999). Simple heuristics that make us smart. New York: Oxford University Press.

Goldstein, D., Gigerenzer, G. (1999). The recognition heuristic: How ignorance makes us smart. In: G. Gigerenzer, P.M. Todd, The ABC Group, Simple heuristics that make us smart (pp. 37-58). New York: Oxford University Press.

Goldstein, D., Gigerenzer, G. (2002). Models of ecological rationality: The recognition heuristic. Psychological Review 109: 75-90.

Hoyer, W.D. (1984). An examination of consumer decision making for a common repeat purchase product. Journal of Consumer Research 11, 822-829. 
lyengar, S.S., Lepper, M.R. (2000). When choice is demotivating: Can one desire too much of a good thing. Journal of Personality and Social Psychology 79, 995-1006.

Malhortra, N.K. (1982). Information load and consumer decision making. Journal of Consumer Research 8: 419-430.

Martignon, L., Hoffrage, U. (1999). Why does one-reason decision making work? A case study in ecological rationality. In: G. Gigerenzer, P.M. Todd, The ABC Group, Simple heuristics that make us smart (pp. 119-140). New York: Oxford University Press.

Nisbett, R.E., Borgida, E. (1975). Attribution and the psychology of prediction. Journal of Personality and Social Psychology 32 (5): 932-943.

Oppenheimer, D.M. (2003). Not so fast! (and not so frugal!) Rethinking the recognition heuristic. Cognition 90: B1-B9.

Payne, J.W., Bettman, J.R., Johnson, E.J. (1993). The adaptive decision maker. Cambridge: Cambridge University Press.

Sahlin, N.-E., Wallin, A., Persson, J. (2010). Decision science: From Ramsey to dual process theories. Synthese 172: 129-143.

Samuels, R., Stich, S., Bishop, M. (2002). Ending the rationality wars: How to make disputes about human rationality disappear. In: R. Elio (ed.), Common sense, reasoning and rationality (pp. 236-268). Oxford: Oxford University Press.

Savage, L.J. (1954). The foundations of statistics. New York: Dover Publications Inc.

Simon, H. (1956). A behavioral model of rational choice. Quarterly Journal of Economics 69: 99-118.

Simon, H. (1990). Invariants of human behavior. Annual Review of Psychology 41: 1-19.

Tversky, A. (1972). Elimination by aspects: A theory of choice. Psychological Review 79: 281-299.

Tversky, A., Kahneman, D. (1974). Judgment under uncertainty: Heuristics and biases. Science 185: 1124-1131.

Wallin, A. (2007). Explanation and environment: The case of psychology. In: J. Persson, P. Ylikoski (eds.), Rethinking Explanation (pp. 163-176). Boston Studies in the Philosophy of Science. Dordrecht: Springer Verlag.

Wallin, A. (2008). Decision making in everyday life. In: P. Gärdenfors, A. Wallin (eds.). A Smorgasbord of Cognitive Science (pp. 249-264). Nora: Nya Doxa.

Wallin, A. (2013). A peace treatise for the rationality wars? External validity and its relation to normative and descriptive theories of rationality. Theory and Psychology 23 (4): 458-478. 